\title{
Constraint Programming Based Biomarker Optimization
}

\author{
Manli Zhou, ${ }^{1,2}$ Youxi Luo, ${ }^{1,3}$ Guoquan Sun, ${ }^{1}$ Guoqin Mai, ${ }^{1}$ and Fengfeng Zhou \\ ${ }^{1}$ Shenzhen Institutes of Advanced Technology, Chinese Academy of Sciences, Shenzhen, Guangdong 518055, China \\ ${ }^{2}$ Shenzhen College of Advanced Technology, University of Chinese Academy of Sciences, Beijing 100049, China \\ ${ }^{3}$ School of Science, Hubei University of Technology, Wuhan, Hubei 430068, China
}

Correspondence should be addressed to Fengfeng Zhou; fengfengzhou@gmail.com

Received 28 September 2014; Accepted 13 December 2014

Academic Editor: Antonello Merlino

Copyright (C) 2015 Manli Zhou et al. This is an open access article distributed under the Creative Commons Attribution License, which permits unrestricted use, distribution, and reproduction in any medium, provided the original work is properly cited.

\begin{abstract}
Efficient and intuitive characterization of biological big data is becoming a major challenge for modern bio-OMIC based scientists. Interactive visualization and exploration of big data is proven to be one of the successful solutions. Most of the existing feature selection algorithms do not allow the interactive inputs from users in the optimizing process of feature selection. This study investigates this question as fixing a few user-input features in the finally selected feature subset and formulates these userinput features as constraints for a programming model. The proposed algorithm, fsCoP (feature selection based on constrained programming), performs well similar to or much better than the existing feature selection algorithms, even with the constraints from both literature and the existing algorithms. An fs CoP biomarker may be intriguing for further wet lab validation, since it satisfies both the classification optimization function and the biomedical knowledge. fsCoP may also be used for the interactive exploration of bio-OMIC big data by interactively adding user-defined constraints for modeling.
\end{abstract}

\section{Introduction}

Biological big data is being accumulated at an accelerated speed, facilitated by the rapid invention and development of bio-OMIC data production technologies $[1,2]$. Interactive exploration technology is widely used to mine knowledge from various big data areas [3] and may be useful to rapidly and accurately detect phenotype-associated biomarkers from the huge amount of bio-OMIC data [4]. This is usually formulated as the feature selection problem $[5,6]$.

Various algorithms have been proposed to choose a few from a large number of features, by optimizing a phenotypic measurement. The principle of parsimony prefers a minimum number of features for an accurate representation of the data [7]. Detailed introduction may be found for both general feature selection algorithms [8] and phenotype-associated biomarker detection algorithms [9] from the literature. Considering millions or more of bio-OMIC features for each sample, although the exhaustive search guarantees the detection of optimal feature subset, its computational requirement exceeds the capacity of any high-performance computing systems under the current parallel computing architecture.
So all the existing feature selection algorithms screen for the suboptimal solutions based on some heuristic rules.

Heuristic feature selection algorithms may be grouped as two classes based on how they generate the finally chosen features. The class I wrapper or group optimization algorithms evaluate a feature subset by testing its classification performance with a learning algorithm. The features are selected by heuristic rules or randomly, and only the feature subset with the best classification performance will be kept for further investigation, for example, forward stepwise selection [10] and ant colony optimization [11]. The class II filtering or individual ranking algorithms measure each feature's correlation with the class labels and rank the features by their measurement. A heuristic assumption is that the combination of top-ranked $K$ features should produce a good classification performance, where $K$ is an arbitrarily chosen integer. They are usually much faster than the class I algorithms but lack model robustness due to the ignorance of feature interdependence [12]. It is also difficult to determine how many features should be chosen from the ordered feature list.

This work proposes a constraint programming based interactive feature selection algorithm, fsCoP, for efficient 
exploration of the bio-OMIC big data. An interactive feature selection problem requires a fast and accurate detection of features and the integration of user-input features in the final result. The majority of existing feature selection algorithms do not consider how to make sure a given feature subset appears among the finally selected features. fsCoP fixes the user-input features in the result by formulating them as constraints of the programming model. Our data show that features chosen by fsCoP perform well similar to or much better than the existing feature selection algorithms in classification, even with the constraints of fixed features from both literature and other algorithms.

\section{Materials and Methods}

2.1. Dataset Downloading and Preprocessing. Two microarray-based gene expression profiling datasets are downloaded from the NCBI GEO database [13]. Both datasets GSE5406 [14] and GSE1869 [15] profiled ischemic cardiomyopathy samples and their controls on the Affymetrix Human Genome U133A Array (HG-U133A) platform. The transcriptomes are normalized using the RMA algorithm [16]. The gene expression profiles of ischemic cardiomyopathy samples and the nonfailing controls are kept for binary classification study in this work.

2.2. Feature Selection Based on Constraint Programming $\left(f_{s} \mathrm{CoP}\right)$. This work proposes a constraint programming based feature selection algorithm, allowing the user to determine a few features in the finally chosen feature subset. The prefixed features may be the biomarkers known to be associated with the phenotype in the literature or the features selected by other feature selection algorithms. This model is proposed to answer the biological questions like whether a few genes together with the ischemic cardiomyopathy associated ACE2 (angiotensin-converting enzyme-2) may constitute an accurate model for the disease early detection. The majority of the existing feature selection algorithms do not have the integrating component for fixing a few features in the final feature subset. Let FixedSubset be the set of features to be fixed in the final result and let $c$ be the class number. Class $j$ has $n_{j}$ samples, where $j=1,2, \ldots, c$. The programming model is defined as follows:

$$
\begin{gathered}
\min _{w_{i}, \xi_{k}} \quad\left\{\sum_{i=1}^{p} w_{i}+\lambda\left(\sum_{j=1}^{c} \sum_{k=1}^{n_{j}} \xi_{k}\right)\right\} \\
\text { s.t. } \quad \sum_{i=1}^{p}\left|s_{k i}^{j}-m_{i}^{j}\right| w_{i}-\xi_{k}<\sum_{i=1}^{p}\left|s_{k i}^{j}-m_{i}^{\sim}\right| w_{i}, \\
\quad \text { for } k \in\left\{1,2, \ldots, n_{j}\right\}, \quad j \in\{1,2, \ldots, c\}, \\
\quad w_{f} \geq \operatorname{MinWeight,} \text { for } f \in \text { FixedSubset, } \\
0 \leq w_{i} \leq 1, \\
\xi_{k} \geq 0 .
\end{gathered}
$$

The average value of the $i$ th feature is denoted as $m_{i}^{j}$ for the samples in class $j$. Formula (2) makes that the centroid of class $j$ is the closest centroid to the samples of the class $j$. Each prefixed feature has the weight no smaller than MinWeight. Each feature has a weight $w_{i} \in[0,1]$, where only features with positive weights are selected by the algorithm.

2.3. Classification Performance Measurements. A binary classification model is trained over the datasets of positive and negative samples, whose numbers are $P$ and $N$, respectively. The classification performance is usually measured by the sensitivity $\mathrm{Sn}=\mathrm{TP} /(\mathrm{TP}+\mathrm{FN})$ and specificity $\mathrm{Sp}=\mathrm{TN} /(\mathrm{TN}+$ $\mathrm{FP}$ ), where $\mathrm{TP}, \mathrm{FN}, \mathrm{TN}$, and $\mathrm{FP}$ are the numbers of true positives, false negatives, true negatives, and false positives. The overall classification performances may be measured by the overall accuracy Acc $=(\mathrm{TP}+\mathrm{TN}) /(\mathrm{TP}+\mathrm{FN}+\mathrm{TN}+\mathrm{FP})$ and balanced overall accuracy Avc $=(\mathrm{TP}+\mathrm{TN}) /(\mathrm{TP}+\mathrm{FN}+$ $\mathrm{TN}+\mathrm{FP})$. Matthew's Correlation Coefficient is also calculated to measure how well a classification model is, and it is defined as $\mathrm{MCC}=(\mathrm{TP} \times \mathrm{TN}-\mathrm{FP} \times \mathrm{FN}) / \operatorname{sqrt}((\mathrm{TP}+\mathrm{FP}) \times(\mathrm{TP}+\mathrm{FN}) \times$ $(\mathrm{TN}+\mathrm{FP}) \times(\mathrm{TN}+\mathrm{FN}))$, where $\operatorname{sqrt}(x)$ is the squared root of $x$.

Fivefold cross validation (5FCV) strategy is used to train the model and calculate how well a model performs. Fluctuation may occur for different seeds of the random number generator. So 30 runs of the 5FCV experiments are carried out with different random seeds.

2.4. Comparison with Four Feature Selection Algorithms. The proposed feature selection algorithm fsCoP is compared with two ranking algorithms, that is, $t$-test (TRank) [17] and Wilcoxon test (WRank) [18], and two other widely used algorithms, that is, prediction analysis of microarrays (PAM) [19] and regularized random forest (RRF) [20].

The ultimate goal of the proposed model is to select a subset of features with accurate classification performance. The performance of a given feature subset is measured by five widely used classification algorithms, including support vector machine (SVM) [21], Naive Bayesian [22], decision tree (DTree) [23], Lasso [24], and $K$-nearest neighbor [25]. The classification model with the best Matthew's Correlation Coefficients is kept for the comparison study.

This work uses the default parameters of all the investigated algorithms implemented in the statistical software $\mathrm{R} /$ Rstudio version 3.1.1 released on July 10, 2014 [26, 27]. A classification model is usually obtained by trying multiple classification algorithms $[28,29]$. So this work compares the feature selection algorithms based on the highest MCC values of the five aforementioned classification algorithms.

\section{Results and Discussion}

3.1. Constrains from the Literature. The angiotensin-converting enzyme-2 (ACE2) at the location Xp22.2 of the human genome HG19 is chosen to be fixed in the algorithm fsCoP, denoted as fsCoP(ACE2). ACE2 was observed to be differentially expressed between ischemic and nonischemic cardiomyopathy and may play a role in transducing the signal of heart failure pathophysiology [15]. The expression 
TABLE 1: Performance comparison of the algorithm fsCoP. fsCoP has no prefixed features, and the model fsCoP(ACE2) has two predetermined features.

\begin{tabular}{|c|c|c|c|c|c|}
\hline \multicolumn{6}{|c|}{ GSE5406 } \\
\hline fsCoP & Sn & Sp & Acc & Avc & MCC \\
\hline SVM & 1.000 & 1.000 & 1.000 & 1.000 & 1.000 \\
\hline NBayes & 1.000 & 0.998 & 1.000 & 0.999 & 0.999 \\
\hline DTree & 0.992 & 0.800 & 0.967 & 0.896 & 0.848 \\
\hline Lasso & 0.999 & 0.900 & 0.987 & 0.950 & 0.939 \\
\hline $\mathrm{KNN}$ & 1.000 & 0.871 & 0.983 & 0.936 & 0.923 \\
\hline fsCoP(ACE2) & Sn & $S p$ & Acc & Avc & MCC \\
\hline SVM & 1.000 & 0.996 & 0.999 & 0.998 & 0.998 \\
\hline NBayes & 1.000 & 0.998 & 1.000 & 0.999 & 0.999 \\
\hline DTree & 0.993 & 0.796 & 0.967 & 0.894 & 0.847 \\
\hline Lasso & 1.000 & 0.907 & 0.988 & 0.953 & 0.944 \\
\hline $\mathrm{KNN}$ & 0.999 & 0.860 & 0.982 & 0.930 & 0.916 \\
\hline \multicolumn{6}{|c|}{ GSE1869 } \\
\hline fsCoP & Sn & $\mathrm{Sp}$ & Acc & Avc & MCC \\
\hline SVM & 1.000 & 0.955 & 0.983 & 0.978 & 0.965 \\
\hline NBayes & 1.000 & 0.972 & 0.990 & 0.986 & 0.979 \\
\hline DTree & 0.907 & 0.000 & 0.567 & 0.453 & $\mathrm{NaN}$ \\
\hline Lasso & 0.960 & 0.989 & 0.971 & 0.974 & 0.943 \\
\hline $\mathrm{KNN}$ & 1.000 & 0.994 & 0.998 & 0.997 & 0.996 \\
\hline fsCoP(ACE2) & $\mathrm{Sn}$ & Sp & Acc & Avc & MCC \\
\hline SVM & 1.000 & 0.939 & 0.977 & 0.970 & 0.953 \\
\hline NBayes & 1.000 & 1.000 & 1.000 & 1.000 & 1.000 \\
\hline DTree & 0.987 & 0.000 & 0.617 & 0.493 & $\mathrm{NaN}$ \\
\hline Lasso & 0.990 & 0.967 & 0.981 & 0.978 & 0.962 \\
\hline KNN & 1.000 & 1.000 & 1.000 & 1.000 & 1.000 \\
\hline
\end{tabular}

TABLE 2: Running time of fsCoP and fsCoP(ACE2) on GSE5406. All the running times are calculated in seconds and column "repeat" gives the number of repeats of each model with different random seed.

\begin{tabular}{lcccc}
\hline Repeat & fsCoP & Avg (fsCoP) & fsCoP(ACE2) & Avg (fsCoP(ACE2)) \\
\hline 5 & 11.95 & 2.39 & 11.78 & 2.36 \\
10 & 23.83 & 2.38 & 23.96 & 2.40 \\
50 & 120.01 & 2.40 & 117.79 & 2.36 \\
100 & 240.23 & 2.40 & 236.75 & 2.37 \\
\hline
\end{tabular}

level of ACE2 is detected by two probe sets (219962_at and 222257_s_at) in the Affymetrix microarray platform U133A (GPL96). These two features will be fixed in the feature subset fsCoP(ACE2), and the performances of the five classification algorithms are compared using the selected features by fsCoP and fsCoP(ACE2).

Firstly, fsCoP and fsCoP(ACE2) achieve similarly good performance on the two investigated datasets, that is, GSE5406 and GSE1869. Table 1 shows that, except the decision tree algorithm on the dataset GSE1869, there are no greater than 0.021 differences in MCC between the two versions of fsCoP. The greatest difference occurs for the NBayes classification algorithm on the dataset GSE1869, where fsCoP(ACE2) (1.000 in MCC) improves fsCoP (0.979).

Secondly, if only the best classification algorithm is chosen for each subset of selected features, fsCoP(ACE2) also performs well similar with fsCoP. SVM(fsCoP) only improves NBayes(fsCoP(ACE2)) by 0.001 in MCC. The other classification performance measurements also show that this is a minor improvement, with the maximal difference being 0.002 in specificity (Sp). The comparison of the best classification models between the two datasets in Table 1 also shows that NBayes(fsCoP(ACE2)) even performs 0.002 better than KNN(fsCoP) on the dataset GSE1869.

fsCoP runs fast similar with or without fixing a few features. The running time of the algorithm fsCoP with or without fixing user-selected features is compared between fsCoP and fsCoP(ACE2). Since fsCoP runs very fast, we repeat the model testing for multiple times with different random seeds, as in Table 2. The data suggests that fsCoP(ACE2) runs slightly faster than fsCoP for most of the times, except for the case of 10 repeats. 


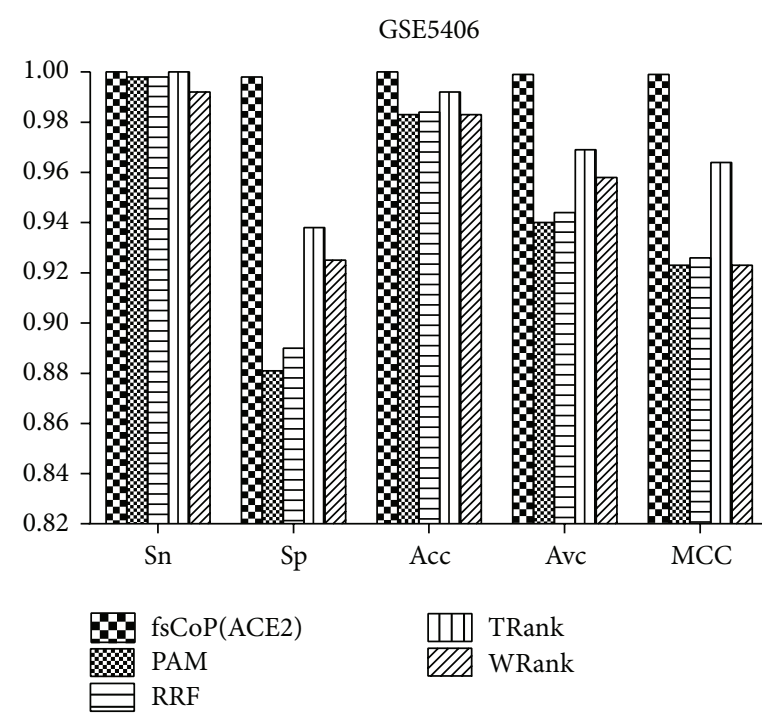

(a)

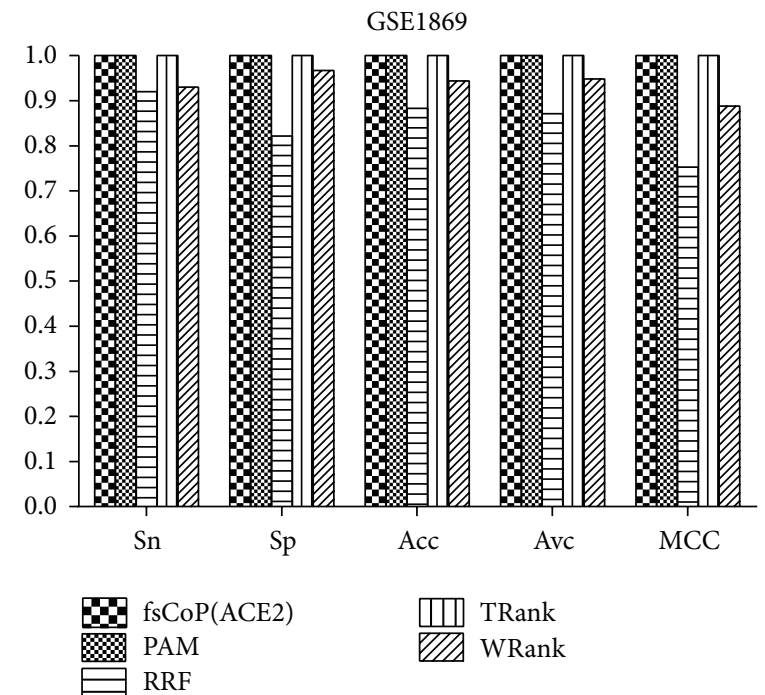

(b)

FIGURE 1: Classification performance comparison of the five feature selection algorithms on the datasets: (a) GSE5406 and (b) GSE1869. The histograms give the detailed values of the classification performance measurements, that is, Sn, Sp, Acc, Avc, and MCC.

3.2. Comparison of $f_{s} \mathrm{CoP}(A C E 2)$ with the Existing Feature Selection Algorithms. A further comparison of fsCoP(ACE2) with the other existing feature selection algorithms is conducted for the best classification algorithms on each of the selected features, as shown in Figure 1. First of all, fs CoP(ACE2) performs the best (100\%) in sensitivity (Sn) with the classification algorithm NBayes on both datasets, as in Figures 1(a) and 1(b). SVM(TRank) achieves the same sensitivities for both datasets, and $\mathrm{KNN}(\mathrm{PAM})$ also achieves $100 \%$ in Sn on the dataset GSE1869. NBayes(fsCoP(ACE2)) achieves 0.998 in specificity (Sp) on the dataset GSE5406, and no other feature selection algorithms reach the same specificity level. Figure 1(a) suggests that the second best feature selection algorithm may be TRank, which achieves 0.964 in MCC on the dataset GSE5406.

3.3. Constraints from the Existing Feature Selection Algorithms. Except for the features selected by TRank, fsCoP improves all the other three feature selection algorithms. fs CoP(A) is defined to be feature list selected by fsCoP, with the fixed features selected by Algorithm A. Figure 2 shows that fs CoP(TRank) achieves the same classification performance as TRank, and, for the three other feature selection algorithms, fs $\mathrm{CoP}()$ achieves higher averaged values and smaller standard deviations for all the five classification performance measurements. The most significant improvement of fs $\mathrm{CoP}$ is observed for the RRF algorithm, with 0.0916 in Sp improvement. So besides the integration of known biomarkers from the literature, fsCoP may also be used to further refine the feature subset selected by the existing feature selection algorithms. Better classification performance with smaller fluctuation may be obtained stably by fsCoP, compared with the algorithms.

After the further refining by fsCoP, features selected by all the four feature selection algorithms achieve $100 \%$ in

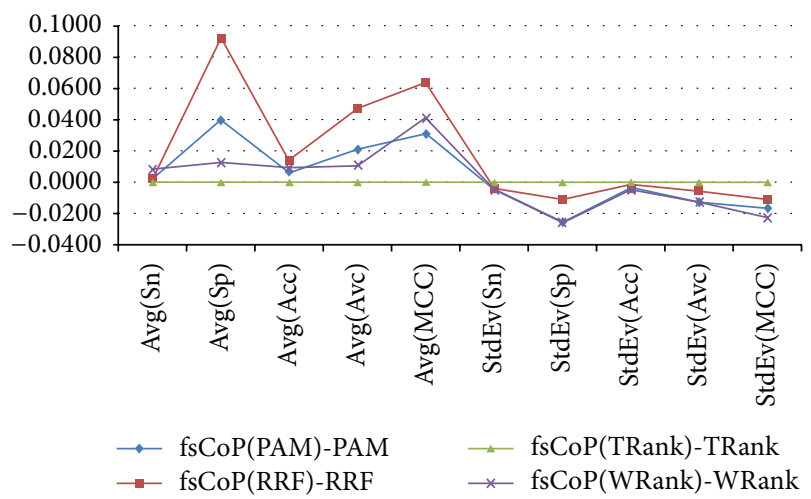

FIGURE 2: Improvements of fsCoP compared with the four investigated feature selection algorithms, by fixing the features selected by each algorithm. The average "Avg()" and standard deviation "StdEv()" of the five classification performance measurements, that is, Sn, Sp, Acc, Avc, and MCC, are calculated over the 30 runnings of 5 -fold cross validations of a given feature subset.

the classification sensitivity, while maintaining at least $92 \%$ in specificity. And at least 0.95 in MCC is achieved for all the four cases.

\section{Conflict of Interests}

The authors declare that there is no conflict of interests regarding the publication of this paper.

\section{Authors' Contribution}

Manli Zhou and Youxi Luo contributed equally to this work. 


\section{Acknowledgments}

This work was supported by the Strategic Priority Research Program of the Chinese Academy of Sciences (XDB13040400), Shenzhen Peacock Plan (KQCX20130628112914301), Shenzhen Research Grants (ZDSY20120617113021359, CXB201104220026A, and JCYJ20130401170306884), the MOE Humanities Social Sciences Fund (no. 13YJC790105) and Doctoral Research Fund of HBUT (no. BSQD13050), and Key Laboratory of Human-Machine-Intelligence Synergic Systems, Chinese Academy of Sciences. Computing resources were partly provided by the Dawning supercomputing clusters at SIAT CAS. The constructive comments from the two anonymous reviewers are greatly appreciated.

\section{References}

[1] E. L. van Dijk, H. Auger, Y. Jaszczyszyn, and C. Thermes, "Ten years of next-generation sequencing technology," Trends in Genetics, vol. 30, no. 9, pp. 418-426, 2014.

[2] N. Chen, W. Sun, X. Deng et al., "Quantitative proteome analysis of HCC cell lines with different metastatic potentials by SILAC," Proteomics, vol. 8, no. 23-24, pp. 5108-5118, 2008.

[3] J. Heer and S. Kandel, "Interactive analysis of big data," XRDS: Crossroads, vol. 19, no. 1, pp. 50-54, 2012.

[4] H. Ge, A. J. M. Walhout, and M. Vidal, "Integrating "omic" information: a bridge between genomics and systems biology," Trends in Genetics, vol. 19, no. 10, pp. 551-560, 2003.

[5] S. Datta and V. Pihur, "Feature selection and machine learning with mass spectrometry data," Methods in Molecular Biology, vol. 593, pp. 205-229, 2010.

[6] Y. Saeys, I. Inza, and P. Larrañaga, "A review of feature selection techniques in bioinformatics," Bioinformatics, vol. 23, no. 19, pp. 2507-2517, 2007.

[7] D. A. Bell and H. Wang, "A formalism for relevance and its application in feature subset selection," Machine Learning, vol. 41, no. 2, pp. 175-195, 2000.

[8] I. Iguyon and A. Elisseeff, "An introduction to variable and feature selection," Journal of Machine Learning Research, vol. 3, pp. 1157-1182, 2003.

[9] S. Baek, C.-A. Tsai, and J. J. Chen, "Development of biomarker classifiers from high-dimensional data," Briefings in Bioinformatics, vol. 10, no. 5, pp. 537-546, 2009.

[10] S. Colak and C. Isik, "Feature subset selection for blood pressure classification using orthogonal forward selection," in Proceedings of the IEEE 29th Annual Northeast Bioengineering Conference, pp. 122-123, IEEE, March 2003.

[11] J. Yang and V. Honavar, "Feature subset selection using a genetic algorithm," in Feature Extraction, Construction and Selection, pp. 117-136, Springer, 1998.

[12] I. A. Gheyas and L. S. Smith, "Feature subset selection in large dimensionality domains," Pattern Recognition, vol. 43, no. 1, pp. 5-13, 2010.

[13] T. Barrett, S. E. Wilhite, P. Ledoux et al., "NCBI GEO: archive for functional genomics data sets-update," Nucleic Acids Research, vol. 41, no. D1, pp. D991-D995, 2013.

[14] S. Hannenhalli, M. E. Putt, J. M. Gilmore et al., "Transcriptional genomics associates FOX transcription factors with human heart failure," Circulation, vol. 114, no. 12, pp. 1269-1276, 2006.
[15] M. M. Kittleson, K. M. Minhas, R. A. Irizarry et al., "Gene expression analysis of ischemic and nonischemic cardiomyopathy: shared and distinct genes in the development of heart failure," Physiological Genomics, vol. 21, pp. 299-307, 2005.

[16] R. A. Irizarry, B. Hobbs, F. Collin et al., "Exploration, normalization, and summaries of high density oligonucleotide array probe level data," Biostatistics, vol. 4, no. 2, pp. 249-264, 2003.

[17] P. Baldi and A. D. Long, "A Bayesian framework for the analysis of microarray expression data: regularized $t$-test and statistical inferences of gene changes," Bioinformatics, vol. 17, no. 6, pp. 509-519, 2001.

[18] W.-M. Liu, R. Mei, X. Di et al., "Analysis of high density expression microarrays with signed-rank call algorithms," Bioinformatics, vol. 18, no. 12, pp. 1593-1599, 2002.

[19] R. Tibshirani, T. Hastie, B. Narasimhan, and G. Chu, "Diagnosis of multiple cancer types by shrunken centroids of gene expression," Proceedings of the National Academy of Sciences of the United States of America, vol. 99, no. 10, pp. 6567-6572, 2002.

[20] H. T. Deng and G. Runger, "Feature selection via regularized trees," in Proceedings of the IEEE International Joint Conference on Neural Networks (IJCNN '12), pp. 1-8, Brisbane, Australia, June 2012.

[21] S. Amari and S. Wu, "Improving support vector machine classifiers by modifying kernel functions," Neural Networks, vol. 12, no. 6, pp. 783-789, 1999.

[22] G. H. John and P. Langley, "Estimating continuous distributions in Bayesian classifiers," in Proceedings of the Eleventh conference on Uncertainty in artificial intelligence: 1995, pp. 338-345, Morgan Kaufmann, 1995.

[23] S. R. Safavian and D. Landgrebe, "A survey of decision tree classifier methodology," IEEE Transactions on Systems, Man, and Cybernetics, vol. 21, no. 3, pp. 660-674, 1991.

[24] D. Ghosh and A. M. Chinnaiyan, "Classification and selection of biomarkers in genomic data using LASSO," Journal of Biomedicine and Biotechnology, vol. 2005, no. 2, pp. 147-154, 2005.

[25] M.-L. Zhang and Z.-H. Zhou, "A k-nearest neighbor based algorithm for multi-label classification," in Proceedings of the IEEE International Conference on Granular Computing, vol. 2, pp. 718-721, Beijing, China, July 2005.

[26] R: A Language and Environment for Statistical Computing, http://www.r-project.org/.

[27] J. S. Racine, "RSTUDIO: a platform-independent IDE for R and sweave," Journal of Applied Econometrics, vol. 27, no. 1, pp. 167172, 2012.

[28] F. Zhou and Y. Xu, "cBar: a computer program to distinguish plasmid-derived from chromosome-derived sequence fragments in metagenomics data," Bioinformatics, vol. 26, no. 16, pp. 2051-2052, 2010.

[29] P. Guo, Y. Luo, G. Mai et al., "Gene expression profile based classification models of psoriasis," Genomics, vol. 103, no. 1, pp. 48-55, 2014. 

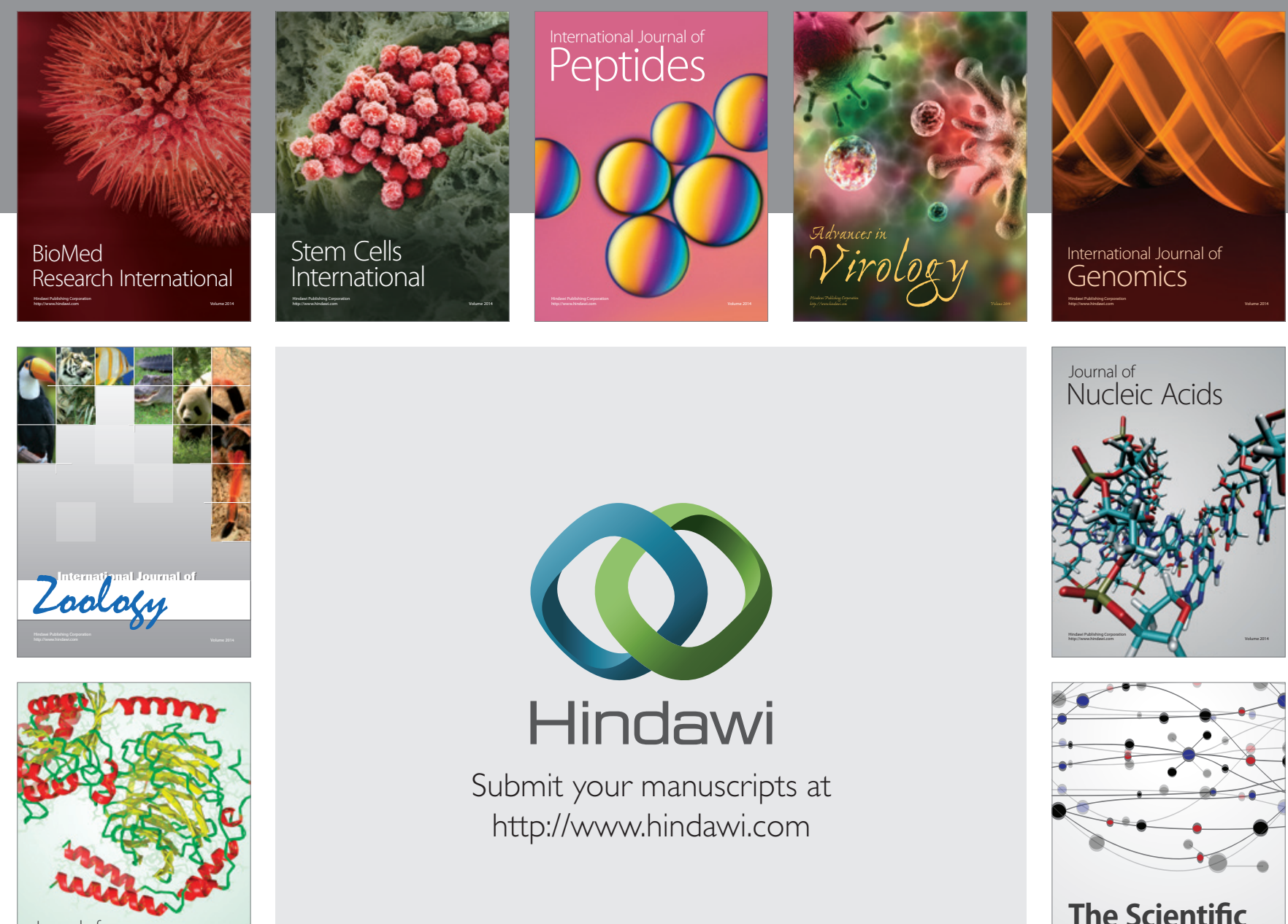

Submit your manuscripts at

http://www.hindawi.com

Journal of
Signal Transduction
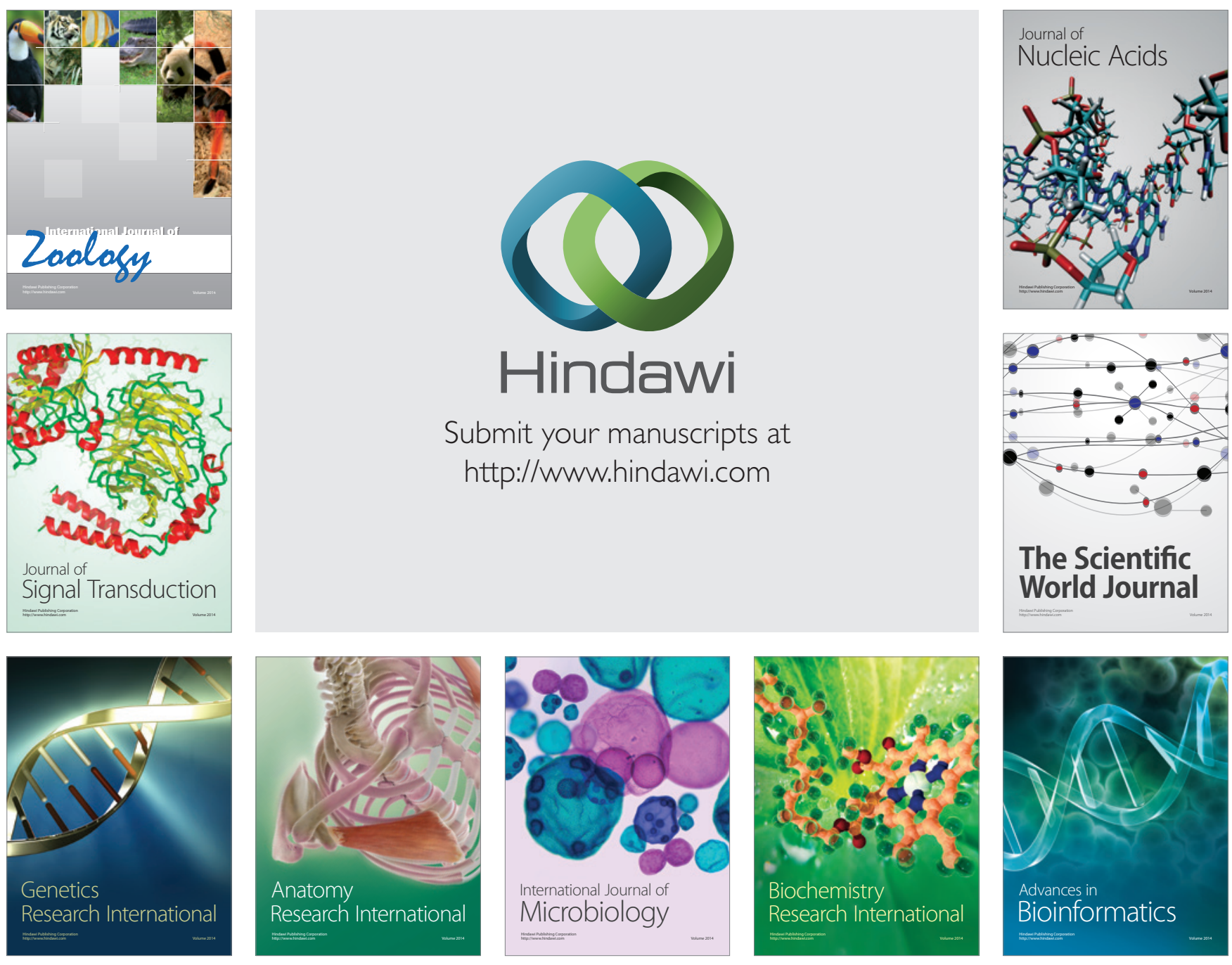

The Scientific World Journal
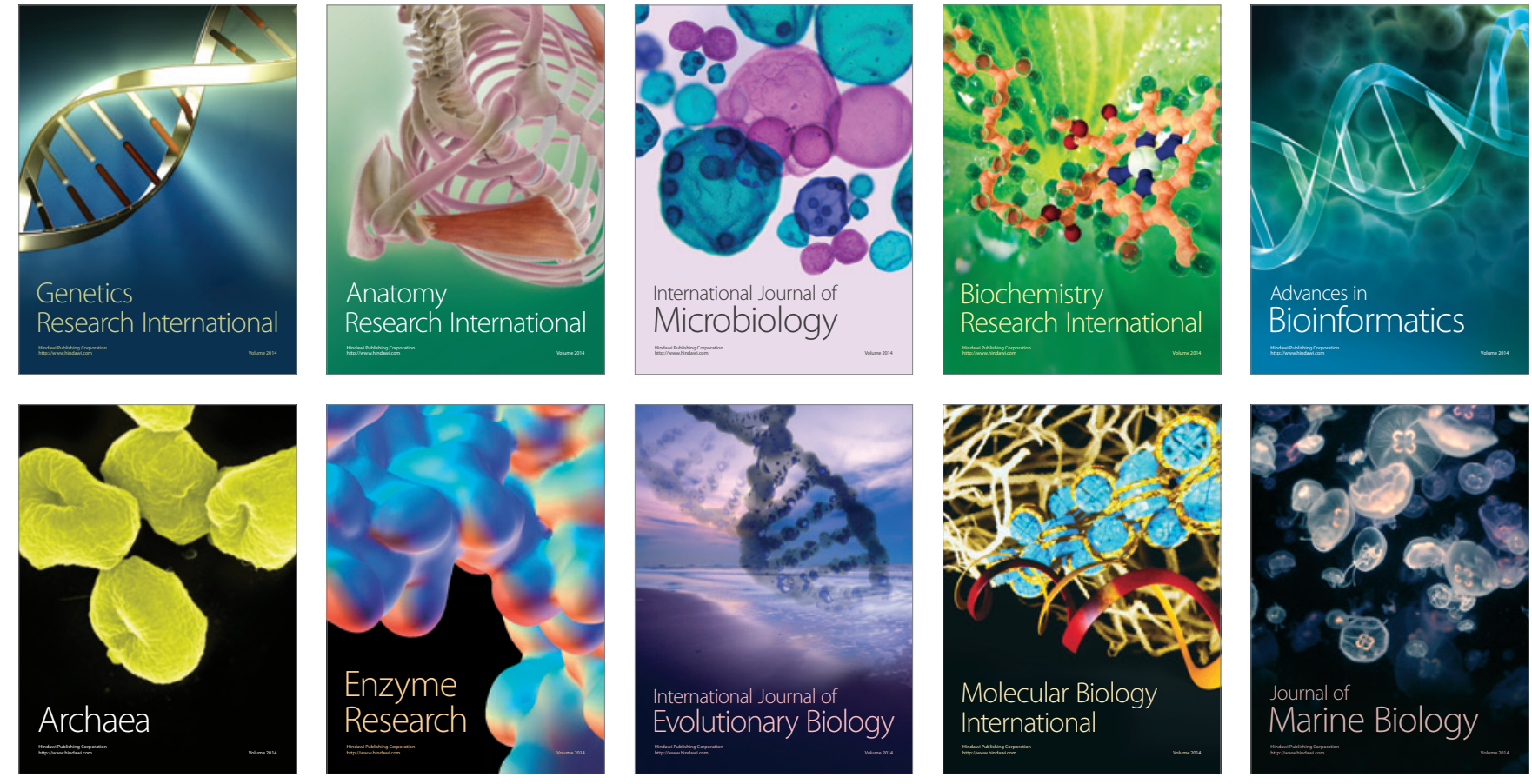